\title{
Product Driven Systems facing unexpected perturbations: how Operational Research models and approaches can be useful?
}

\author{
Alexis Aubry ${ }^{1,2}$, Hind Bril ${ }^{1,2}$, André Thomas ${ }^{1,2}$, Mireille Jacomino ${ }^{3}$ \\ ${ }^{1}$ Université de Lorraine, CRAN, UMR 7039, Campus Sciences, BP 70239, Vandœuvre-lès- \\ Nancy Cedex, 54506, France \\ ${ }^{2}$ CNRS, CRAN, UMR 7039, France \\ \{alexis.aubry, hind.el-haouzi, andre.thomas\}@univ-lorraine.fr \\ ${ }^{3}$ Univ. Grenoble Alpes, CNRS, G-SCOP Laboratory, 38000 Grenoble France \\ mireille.jacomino@grenoble-inp.fr
}

\begin{abstract}
Production planning and control and more generally taking a decision in the context of production systems often consider that input information are known, static and predictable. However, uncertainties on data and perturbations are recorded in the genetic of every production system. For instance, it is impossible to know exactly the level of the demand for a product, the availability of resources, etc. Dealing with this issue asks the question of the ability to take robust decisions against uncertainty (off-line) or the ability to be flexible (on-line). This paper proposes to analyze how Product Driven Systems - as reactive systems against unpredicted perturbations - can be part of operational research solution process against perturbations. Moreover, an overview of models and approaches for dealing with uncertainty in Operational Research is given and a first proposition is made for applying these elements into PDS as decision-makingagainst-perturbations engines.
\end{abstract}

Keywords: Production Planning and Control; Product Driven Systems; Uncertainty; Robustness; Flexibility; Operational Research

\section{$1 \quad$ Introduction}

Since some years, Product Driven Systems (PDS) are becoming a credible way to control product flows on shop-floors and in supply chains thanks to Auto-ID and especially RFID technologies. PDS are defined as systems in which products are able to be active and are at the core of an enterprise architecture which integrates every actor in the company, from the central systems to the processes, products and also operators, into the same ambient information system [1]. The Intelligent Product (IP) paradigm $[2,3]$ was a step further to the use of RFID-tagged products, and not only for identification objectives. IP has demonstrated the efficiency of speeding up and making more visible information flows in logistic operations [4]. Following, a new interpretation of 
the stigmergy concept was put forward in [1], where the cooperation between production actors (products, resources and all used manufacturing appliances) was achieved thanks to attributes called informational pheromones and carried by RFID-tagged products. Products collaboration is a concept on which many researchers $[5,6]$ have focused their works.

Thus, this Product Driven Systems (PDS) paradigm has been established and its relevance has been highlighted precisely to deal with new product flows control approaches, especially to react to unpredicted events occurring on the shop-floors and in the supply chains. Indeed, anybody with production or logistic experience knows that, often, a predicted plan or schedule cannot be exactly executed! Events occurring on the shop-floor, or in the supply chain lead to uncertainties in daily activities inducing needs for adjustments in plans. That is one of the main reasons that justify PDS.

On the other hand, in the previous decades, a lot of research teams have proposed a huge of models, built on exact approaches or on heuristics, useful to schedule or reschedule production and logistic activities. In the design or management of these various types of control systems, and particularly for production and logistic ones, a number of variables have to be frequently determined so as to optimize a given performance criterion. Optimization models are used to search for the best possible values of a vector of variables (e.g. number of resources, production planning in a manufacturing system and so on). In this context, operational research approaches are privileged approaches when dealing with optimization in production systems. However, in most dedicated works, the optimization model is built from data that characterize given and fixed environmental conditions (e.g., assumed nominal processing times, fixed transportation delays, exact amount of demand etc). However, in practice, perturbations can modify these values, and thus change the data that were initially assumed to be known and static. The expected values (also called reference instance in [7] or base environmental scenario in [8]) are supposed to model the environment that the domain expert think relevant to characterize the actual operating conditions of the studied system. In practice, good or even optimal performances, achieved by the system in given conditions can be drastically deteriorated if these conditions change. For instance, a delivery delay, evaluated through an optimization model, can be considered as acceptable, with regards to the customer demand, if breakdowns occur at a given rate, but can become unsatisfactory if the breakdown rate increases. To deal with this issue, it is necessary to take the data uncertainty and the perturbations into account. Since several years, operational researchers are aware of this issue and have proposed some process and approaches to deal with uncertainty and perturbations.

The goal of this paper is to analyze how the PDS paradigm can be involved inside the process proposed by operational researchers to deal with perturbations and to identify the knowledge and information about operational research works dealing with scheduling under uncertainties and to highlight if some of them could be useful to deal with PDS re-scheduling acting in uncertain production and/or logistic environment. The rest of the paper is organized as follows. The section 2 is dedicated to present how optimization can take into account inherent uncertainties and perturbations and proposes a 3-steps solution process to deal with uncertainties and perturbations. The sec- 
tion 3 deals with the step 1 and more specifically on the concepts of robustness in operational research. The section 4 focuses on the step 2 and presents how the concept of Product-Driven-System can be a good candidate for implementing this step as a reacting system against perturbations. Moreover, this section proposes a first overview of the models and approaches coming from Operation Research that can be useful for implementing efficiently these types of system. Finally, a conclusion is given with some perspectives in the section 5 .

\section{Dealing with uncertainty: a full solution process}

\subsection{Classical optimization}

Classically, solving an optimization problem consists in building a solution $S$ that optimizes a criterion $z$ (that is assumed to be a minimization criterion in this section without loss of generality) and satisfies some constraints assuming that the problem data are certain and sure. This particular string of data can be seen as a forecast instance $I^{r e f}$ that is used to compute $S$. Getting an optimal solution is still a problem that is often hard. An optimal solution to the problem for an instance $I$ is denoted $S_{I}^{*}$ and its corresponding performance is denoted $z_{I}^{*}$. Otherwise, the performance of a solution $S$ applied to an instance $I$ relatively to an optimization criterion $z$ is denoted $z_{I}(S)$.

The classical way to solve an optimization problem without uncertainty is the predictive approach. An off-line algorithm builds an optimal solution $S_{I^{r e f}}^{*}$ for the forecast instance $I^{r e f}$, and guarantees an optimal performance for this instance only, valued by $z_{I^{r e f}}^{*}$. In practice, the real system is subject to perturbations such that the solution $S_{I^{r e f}}^{*}$ is applied to the actual instance $I$ that may be different from the forecast instance $I^{r e f}$, and $S_{I^{r} \text { ref }}^{*}$ may even no longer be admissible. In the most optimistic case (when the solution remains admissible for $I$ ), the actual performance $z_{I}\left(S_{I^{r e f}}^{*}\right)$ can be "far" from the forecast performance $z_{I}^{*}$ ref , and also far from the optimal value for $I, z_{I}^{*}$. Then, a costly solution step may return a poor-quality solution.

\subsection{A full solution process for dealing with uncertain context}

There are two complementary ways for taking uncertainties into account. The first one is concerned with uncertainties that are explicitly depicted. Those uncertainties are somehow expected. The second way concerns unexpected perturbations which the system has undergone. Expected uncertainties can be taken into account more or less in an anticipative optimisation structure, that is the robust approach. Unexpected uncertainties have to be taken into account in a reactive procedure. In [9], the generic structure for dealing with uncertainties is addressed. It is formally defined for addressing scheduling problems. It is composed of three steps.

\section{Step 0: static problem definition.}


Classic specifications of the optimisation problem are given along with the optimisation criterion z. Moreover,.when uncertainties are identified, it is necessary to produce a model of these uncertainties. Several possibilities exist. Uncertainties can be modelled as stochastic parameters [10] or parameters belonging to fuzzy intervals [11] or belonging to a set of scenarios or instances [12], [7]. This set of instances can be a continuous or a discrete interval. A specific robustness criterion has to be defined related to the expected risk answering the question: "what must be guaranteed despite these perturbations?"

Step 1: calculation of a set of anticipative solutions by an off-line algorithm.

From the available knowledge about perturbations (the risk to be covered defined in step 0 ), one or more algorithms can be used to build a set of robust solutions guarantying a performance on the considered risk as defined in step 0 . This set can include one or more solutions. Instead of computing an optimal solution for a single forecast instance, a global performance on the risk to be covered is addressed. Such solutions if they exist, are said to be robust for the considered risk.

Step 2: calculation of the applied solution by an on-line algorithm.

The on-line algorithm uses the progressive knowledge about environment to implement a solution knowing the anticipative solutions. This solution can be chosen among the set of solutions calculated in step 1 or can result from a reactive adaptation of anticipative choices. The gap between the implemented solution and the anticipative one depends on the perturbations that occur: if they are included in the expected risk to be covered then the reactive procedure consists in choosing a solution among the robust previously computed solutions, otherwise all changes are possible. Moreover, this asks the question of the detection of the perturbation.

In this dynamic procedure, the problem of guaranteed performance is open.

\section{$3 \quad$ Step 1: taking robust decisions}

\subsection{What is robustness?}

Developing robustness features for the decision to be made has appeared to be an efficient way of coping with deterministic uncertainties as in [12]; [13,14,15,16] even though researchers use different measures of robustness. [17] identifies several types of uncertainty involved in robustness optimization and several ways of formulating and addressing the corresponding problems in the field of parameter design. In [16], robustness is defined as a capacity for withstanding "vague approximations" and/or "zones of ignorance" to prevent undesirable impacts, notably the degradation of the properties to be maintained. What are exactly the so-called properties to be maintained and the considered "approximations" and "zones of ignorance" is strongly application dependent, and has led researchers to develop a large variety of robustness approaches.

Many robustness definitions can be found in the literature. 
The five following papers are devoted to such definitions: [12], [15,16], [18,19]. A lot of other papers are devoted to finding a robust solution for a given problem and explicitly proposing or not a measure of robustness. It is possible to sort the robustness definitions into two categories. The first category defines a robust solution as a solution that optimizes a robustness criterion. This point of view has the advantage that classical models and approaches used in Operational Research can be used for addressing this problem. The second category defines a robust solution as a solution that satisfies a condition (or a set of conditions). Such a vision for robustness allows practitioners to keep their solution processes and to check for the robustness of any solution with no restriction.

\section{Step 2: React after the occurrence of perturbations}

\subsection{The product centric paradigm/Product Driven Systems}

When perturbations don't concern directly data (like variation in demand) but are relative to specific events occurring on the shop-floor or in the supply chain, it is necessary to react on-line. As previously introduced, Product Driven Systems (PDS) are seen today as a good alternative to address this challenge. The idea is to deal directly with products whose information content is permanently bound to their material content and which are able to influence the decisions made about and for them. Therefore, productdriven control has an impact on decision-making procedures as well as on information exchange and storage. PDS are thus good candidates for covering the step 2.

\subsection{Re-optimization or Online optimization}

In the production step, an in-process product can manage its own manufacturing, dispatching or operating lists and priorities, according to the real state of the production system and possible dysfunctions occurring on the shop floor or modifications concerning the due dates or the demand volume. It can ask for specific production services to resources and communicate with the other products to negotiate priorities and production deadlines. In the logistic phase, active products in a system with safety constraints can supervise their own operations, triggering alarms when required by cooperating with other active components, thus improving the availability and maintainability of the system and obtaining an ambient system [20].

All these re-scheduling cases could be solved in a more reactive way in switching down from the centralize control system to PDS. Very quickly PDS could find, if exist, a feasible solution to do production or logistic activities remaining after the specific event. Obviously if no solution emerges at the operational level it would be still necessary to switch back to the centralize way to find an optimal solution. Ideally, to be the most possible reactive, this kind of process must be "on-line" to be sure that the real system won't change during the decision process as highlighted in [21].

The main issue for researchers is then to provide accurate mechanisms to define "online" the best switching dates (and/or the best switching decision-making levels) for 
control holons/agents so that they behave in a sense that the behavior of the hybrid architecture stays globally optimized despite disturbances. In this context, flexibility and resilience are two performance indicators that are interesting for evaluating a PDS.

\subsection{Models and approaches for reacting after perturbations}

\section{Models.}

Regarding Operational Research, two main models are used for modelling an optimization problem: graphs and mathematical programs. When dealing particularly with operations scheduling problems, it is easy to check that the mathematical programs are more used in this context. According to the type of the mathematical program (linear or not, with integer variables or not...), associated solution methods are existing for finding the optimal solution. And these methods are implemented in commercial tools like Cplex, Lingo. The efficiency of the methods for solving such models is particularly sensitive to the type of variables, the number of variables and the number of constraints.

When dealing with operations scheduling (and so re-scheduling), two subproblems must be solved. The first one concerns the allocation of operations to machines and the second one concerns the sequencing of these operations with possible precedence constraints to be satisfied. We can note that the allocation problem can be sometimes fixed a priori when only one machine is qualified for executing a given operation. We will see in the next paragraph that the fact that the allocation problem is fixed or not has an incidence on the solving method.

\section{Solution approaches.}

Classical Operational Research approaches for solving optimization problems can be split into two categories:

- Exact methods: Branch and Bounds algorithms (for solving Mixed Integer Linear Programs), dedicated algorithms. These approaches have the advantage to be able to find the optimal solution but are often not efficient for real-sized problems.

- Approximation methods: mainly heuristics and metaheuristics. These approaches have the advantage to be efficient even for real-sized examples and to be able to find good solution regarding the optimization criterion.

For identifying the relevant approaches for solving re-scheduling problems in the context of PDS, it is mandatory to take into account at least two inputs: the available time for finding the schedule and the type of decision to be taken.

The available time is clearly dependent of the duration of the unpredicted perturbation. In fact, if the time for taking the decision becomes bigger than the time for solving directly the perturbation, the re-scheduling becomes useless. For instance, imagine that a machine failed and that we can predict that the machine can be repaired with a given duration, this will fix the available time for finding a new schedule. It is precisely here that the PDS concept is the most useful because its application must help to evaluate this available time through the information that can give the product the closest from the production system. The product can help to detect the unpredicted perturbation and 
then the merged information coming from the different products is able to give an overall situation and then can help to decide which type of decision must be taken.

The type of decision to be taken concerns mainly if re-allocation is needed or not. If re-allocation is needed, that means that an integer variable is necessary for representing the allocation of each operation to one machine. That means that the mathematical program for modelling the problem becomes a Mixed Integer Linear Program (MILP). This type of problem is known to be NP-Complete: that means that no efficient solution method is known for solving efficiently a MILP.

According to several inputs, we define a first table giving the preferable solution methods depending on some criteria arbitrarily defined. To our best of knowledge, such a work has never been done such that the identified criteria/needs should be enriched and discussed. The first table is given in the next figure. Future works must be done to i) fill the gaps in the table, ii) better justifying the criteria in the table.

\begin{tabular}{|c|c|c|c|c|c|c|c|c|c|c|}
\hline Solution Approach & Criterion/Need & $\begin{array}{c}\text { Optimality } \\
\text { need }\end{array}$ & \multicolumn{2}{|c|}{$\begin{array}{c}\text { Available time for finding } \\
\text { a feasible solution }\end{array}$} & $\begin{array}{c}\begin{array}{c}\text { Participation of } \\
\text { a decision } \\
\text { expert }\end{array} \\
\end{array}$ & \multirow{2}{*}{$\begin{array}{c}\begin{array}{c}\text { Several } \\
\text { solutions } \\
\text { needed }\end{array} \\
\text { Yes } \\
\text { (population) }\end{array}$} & \multirow[t]{2}{*}{$\begin{array}{c}\text { Fixed or } \\
\text { Flexible route }\end{array}$} & \multirow[t]{2}{*}{$\begin{array}{l}\text { Need of } \\
\text { homogeneity } \\
\text { of the route }\end{array}$} & \multirow{2}{*}{$\begin{array}{c}\begin{array}{c}\text { Possible } \\
\text { splitting or } \\
\text { not }\end{array} \\
\text { Yes }\end{array}$} & \multirow{2}{*}{$\begin{array}{c}\begin{array}{c}\text { Possible } \\
\text { overlapping } \\
\text { or not }\end{array} \\
\text { Yes }\end{array}$} \\
\hline \multirow{6}{*}{ Metaheuristics } & Genetic Algorithm & \multirow{6}{*}{$\begin{array}{l}\text { Feasible } \\
\text { solution } \\
\text { accepted }\end{array}$} & \multirow{6}{*}{$\begin{array}{l}\text { real-time } \\
\text { decision }\end{array}$} & & & & & & & \\
\hline & Simulated Annealing & & & & & & & & & \\
\hline & Tabu Search & & & & & & & & & \\
\hline & $\begin{array}{l}\text { Ant Colony } \\
\text { Optimization }\end{array}$ & & & & & & & Yes & Yes & Yes \\
\hline & $\begin{array}{l}\text { Particle Swarm } \\
\text { Optimization }\end{array}$ & & & & & & & & Yes & Yes \\
\hline & BAT Optimization & & & $\begin{array}{c}\text { Very quick } \\
\text { convergence }\end{array}$ & & & & & & \\
\hline \multirow{2}{*}{ Exact methods } & MILP & \multirow{2}{*}{$\begin{array}{l}\text { Optimal of } \\
\text { feasible } \\
\text { solution }\end{array}$} & & & \multirow{2}{*}{$\begin{array}{c}\text { Process } \\
\text { acceleration }\end{array}$} & & Flexible route & & & \\
\hline & LP & & & & & & Fixed route & & & \\
\hline
\end{tabular}

Fig. 1. - Identified solution methods according to several criteria

\section{Conclusions}

This paper investigates the product-driven systems as a candidate for reacting against perturbations and for taking part in a full solution process existing in the operational research context. Moreover, this paper is a first attempt to identify the solution approaches from operational researchers that are relevant for implementing a PDS.

Valuable efforts should still be done for better identifying the different situations that can occur when dealing with perturbations, and thus to associate the better solution approaches to deal with these situations.

\section{References}

1. Pannequin, R., Thomas, A.: Another Interpretation of Stigmergy for Product-Driven Systems Architecture. Journal of Intelligent Manufacturing, 23(6), 2587-2599 (2011)

2. Wong, C.Y., McFarlane, D., Zaharudin, A.A., Agarwal, V.: The Intelligent Product Driven Supply Chain. In: Proceedings of Systems, Man and Cybernetics, 2002 IEEE International Conference on, vol. 4 (2002) 
3. Morel, G., Panetto, H., Zaremba,M., Mayer, F.: Manufacturing Enterprise Control and Management System Engineering: paradigms and open issues. Annual Reviews in Control, 27(2), 199-209 (2003)

4. Klein, T., Thomas, A.: Opportunities to reconsider decision making processes due to AutoID. International Journal of Production Economics, 121(1), 99-111 (2009)

5. Yoshimura, M.: System Design Optimization for Product Manufacturing. Concurrent Engineering: Research and Applications, 15(4), 329-343 (2007)

6. Wenyan, S., Ming, X., Wang, P.: Collaborative Product Innovation Network: Status Review, Framework, and Technology Solutions. Concurrent Engineering: Research and Applications, 21(1), 55-64 (2013)

7. Espinouse, M.-L., Jacomino, M., Rossi, A.: On the Robustness of Multi-Purpose Machines Workshop Configuration. In: Flexibility and Robustness in Scheduling. ISTE Ltd, London, United Kingdom (2008)

8. Pierreval, H., Durieux-Paris, S.: Robust Simulation with a Base Environmental Scenario. European Journal of Operational Research 182, 783-793 (2007)

9. Billaut, J.-C., Moukrim, A., Sanlaville, E.: Introduction to Flexibility and Robustness in Scheduling. In: Flexibility and Robustness in Scheduling. ISTE Ltd, London, United Kingdom (2008)

10. Dauzère-Pérès, S., Castagliola, P., Lahlou, C.: Service Level in Scheduling. In: Flexibility and Robustness in Scheduling. ISTE Ltd, London, United Kingdom (2008)

11. Dubois, D., Fargier, H. Fortemps, P.: Fuzzy Scheduling: Modelling Flexible Constraints vs. Coping with Incomplete Knowledge. European Journal of Operational Research, 147, 231$252(2003)$

12. Kouvelis, P., Yu, G.: Robust Discrete Optimization and its Applications. Kluwer Academic Publishers, Dordrecht, the Netherlands (1997)

13. Vincke, P.: Robust Solutions and Methods in Decision-Aid. Journal of Multi-Criteria Decision Analysis, 8, 181-187 (1999)

14. Perny, P., Spanjaard, O., Storme, L.X.: A Decision-Theoretic Approach to Robust Optimization in Multivalued graph. Annals of Operations Research, 147, 317-341 (2006)

15. Kalaï, R., Aloulou, M.A., Vallin, P., Vanderpooten, D.: Robust 1-Median location Problem on a Tree. In: Proceedings of the ORP3 (Euro Conference for Young Researchers and Practitionners), Valencia, Spain

16. Roy, B.: Robustness in Operational Research and Decision Aiding: A Multi-Faceted Issue. European Journal of Operational Research, 200(3), 629-638 (2010)

17. Beyer, H.G., Sendhoff, B.: RobustOptimization - a Comprehensive Survey. Computer Methods in Applied Mechanics and Engineering, 196, 3190-3218 (2007)

18. Aubry, A., Rossi, A., Jacomino, M.: A Generic Off-line Approach for Dealing with Uncertainty in Production Systems Optimisation. In: Proceedings of the $13^{\text {th }}$ IFAC Symposium on Information Control Problems in Manufacturing (IFAC-INCOM'09), Moscow, Russia, 1464-1469 (2009)

19. Galand, L., Spanjaard, O.: OWA-based Search in State Space Graphs with Multiple Cost Functions. In: Proceedings of the $20^{\text {th }}$ International Florida Artificial Intelligence Research Society Conference (FLAIRS'07), 86-91 (2007)

20. Parlikad, A., K. McFarlane, D.: RFID-Based Product Information in End-of-life Decision Making. Control Engineering Practice, 15, 1348-1363 (2007)

21. Li, M., Bril El-Haouzi, H., Thomas, A., Guidat, A.: Fuzzy Decision-Making Method for Product Holons Encountered Emergency Breakdown in Product-Driven System: an Industrial Case - SOHOMA'14, Nancy (2014) 